\title{
Not all malignant pleural effusions are created equal
}

\author{
Jeffrey Javidfar, MD
}

From the Division of Cardiothoracic Surgery, Emory University Hospital, Atlanta, Ga.

Disclosures: Author has nothing to disclose with regard to commercial support.

Received for publication June 21, 2018; revisions received June 21, 2018; accepted for publication June 22, 2018.

Address for reprints: Jeffrey Javidfar, MD, Division of Cardiothoracic Surgery, Emory University Hospital, 1365

Clifton Rd NE, Clinic Building A, Room 2219, Atlanta, GA 30322 (E-mail: Jeffrey.Javidfar@emory.edu).

J Thorac Cardiovasc Surg 2018;156:1262

$0022-5223 / \$ 36.00$

Copyright $(\subset) 2018$ by The American Association for Thoracic Surgery

https://doi.org/10.1016/j.jtcvs.2018.06.078

Do we really need more trials studying malignant pleural effusions (MPEs)? We learned how to manage this problem as junior residents, with randomized, controlled trials to help guide our decision making. ${ }^{1,2}$

Such might be the initial response to a call for more careful study of this topic. ${ }^{3}$ Given the high annual incidence of MPEs, studies focusing on their management are plentiful in the recent literature. Such studies pit talc pleurodesis against tunneled indwelling pleural catheters.

The Australian Malignant Pleural Effusion (AMPLE) trial showed that the indwelling pleural catheter cohort spent fewer days in the hospital and required fewer invasive pleural procedures, with no differences in breathlessness or quality of life. ${ }^{1}$ In patients with a limited life expectancy, additional time spent at home with loved ones can become a leading metric, especially if it is associated with fewer invasive procedures. This has contributed to a shift away from surgical or chemical pleurodesis to the outpatient management of MPEs with pleural catheters. Earlier trials may have left out swaths of patients, however, because their particular disease processes did not fit into cookie-cutter studies. We are awaiting the results of newer randomized, controlled trials that have completed enrollment to determine whether these patients were captured. ${ }^{4,5}$

In this issue of the Journal, Demmy ${ }^{3}$ contends that not all MPEs are alike. He says that by clumping together various malignancies and degrees of trapped lung to facilitate recruitment, we inadvertently dull a study's ability to detect therapeutic nuances.

Demmy $^{3}$ provides a synopsis of trials that guide how we manage MPEs today, and expertly highlights the pending questions in the literature. Several of the randomized, controlled trials in this area had challenges with enrollment, and-perhaps as a result of clumping - they did not detect significant differences between the treatment arms. Is this a problem with study design? It becomes apparent that these trials can exclude and understudy patients with trapped lung, because there is a commonly held view that entrapment impairs pleurodesis. As a result, the opportunity to study or control for variables such as quantifiable pleural elasticity is missed. In addition, other issues requiring further examination are identified, such as cost of disposables and patient compliance, newer catheters and pleurodesis protocols, and catheter-related loculations.

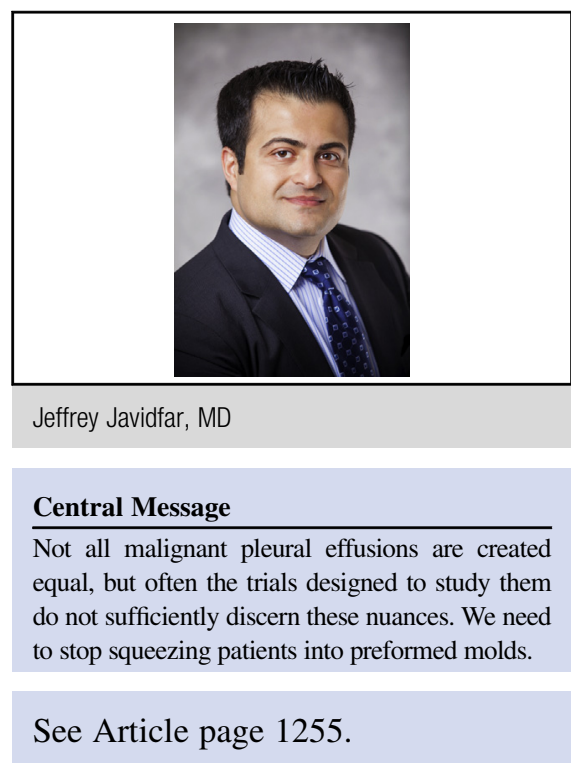

An interesting way to couple the treatments is to combine talc with the indwelling catheter. Bhatnagar and colleagues ${ }^{2}$ showed that outpatient administration of talc through an indwelling pleural catheter in patients without significant trapped lung resulted in improved pleurodesis at 35 days. The addition of talc roughly doubled the pleurodesis rate and resulted in significantly improved quality of life scores, without increasing adverse events. At my institution, we combine video-assisted thoracoscopic surgical talc pleurodesis with simultaneous placement of an indwelling pleural catheter and have retrospectively noticed improved pleurodesis.

The management of MPEs may not be a very controversial topic on its surface, but the aphorism still holds: the devil is in the details... of the study design. ${ }^{3}$ Perhaps we should heed Demmy's call for a new detailed prospective registry that will help us design more meaningful trials.

\section{References}

1. Thomas R, Fysh ET, Smith NA, Lee P, Kwan BC, Yap E, et al. Effect of an indwelling pleural catheter vs talc pleurodesis on hospitalization days in patients with malignant pleural effusion: the AMPLE randomized clinical trial. JAMA. 2017;318:1903-12.

2. Bhatnagar R, Keenan EK, Morley AJ, Kahan BC, Stanton AE, Haris M, et al. Outpatient talc administration by indwelling pleural catheter for malignant effusion. N Engl J Med. 2018;378:1313-22.

3. Demmy TL. Optimizing the study of tunneled intrapleural catheters for malignant pleural effusions. J Thorac Cardiovasc Surg. 2018;156:1255-9.

4. Sivakumar P, Douiri A, West A, Rao D, Warwick G, Chen T, et al. OPTIMUM: a protocol for the multicentre randomised controlled trial comparing out patient talc slurry via indwelling pleural catheter for malignant pleural effusion vs usual inpatient management. BMJ Open. 2016;6:e012795.

5. Azzopardi M, Thomas R, Muruganandan S, Lam DCL, Garske LA, Kwan BCH, et al. Protocol of the Australasian malignant pleural effusion-2 (AMPLE-2) trial: a multicentre randomised study of aggressive versus symptom guided drainage via indwelling pleural catheters. BMJ Open. 2016;6:e011480. 